\title{
Evidence for a further fractionation of the verbal STM system: Selective impairments for item and serial order retention capacities in STM patients
}

\author{
Steve Majerus ${ }^{\text {a,b }}$, Marie-Noëlle Metz-Lutz ${ }^{\mathrm{d}}$, Marie-Anne Van der Kaa ${ }^{\mathrm{c}}$, Martial Van der Linden ${ }^{\mathrm{a}, \mathrm{e}}$, Martine \\ Poncelet ${ }^{\mathrm{a}}$ \\ ${ }^{a}$ Cognitive and Behavioral Neuroscience Research Center, Université de Liège, Boulevard du Rectorat, B33, 4000 Liège, Belgium \\ ${ }^{\mathrm{b}}$ National Fund of Scientific Research, Belgium \\ ${ }^{\mathrm{c}}$ University Hospital, Liège, Belgium \\ ${ }^{\mathrm{d}}$ Université Louis-Pasteur, France \\ University of Geneva, Switzerland
}

\section{Introduction}

Recent studies suggest that verbal short-term memory (STM) is not a unitary system, but relies on a number of distinct capacities. A critical distinction has been proposed between item STM capacity (i.e., the retention of phonological and semantic characteristics of the information to be remembered) and serial order STM capacity (i.e., the sequential order in which the information has been presented). Functional neuroimaging studies suggest that item STM is supported by a network including the left or bilateral temporo-parietal area while serial order STM appears to recruit a network including the right intraparietal sulcus, the premotor frontal cortex and cerebellar cortex (Majerus et al., 2006a). Developmental studies have also shown that item and serial order STM capacity are independent predictors of vocabulary knowledge (in children) and vocabulary learning (in adults), with serial order STM capacity being the most reliable predictor (Majerus, Poncelet, Greffe, \& Van der Linden, 2006b). Moreover, recent computational STM models propose that the specific function of a dedicated STM system is to store serial order information while storage of item information depends on temporary activation of the language system (e.g., Burgess \& Hitch, 2006). The aim of the present multiple case study is to provide neuropsychological evidence for the item/order STM distinction, by presenting three STM patients showing differential levels of impairment for item and order STM.

\section{Participants}

Two patients, TG and DC (age: 21 and 23 years, respectively), had suffered from childhood epileptic aphasia. Although their language functions had largely recovered at the time of this study, they still presented poor verbal STM performance for word list recall and digit span tasks (see Table 1). Brain imaging performed during adolescence and adulthood had shown abnormal activation in bilateral temporo-parietal areas (patient TG) and abnormal activation in the left temporo-parietal area and the right intraparietal sulcus (patient DC). A third patient, AP (age: 71 years) had suffered from a stroke in the left posterior peri-sylvian area in 2004; his symptoms at the time of this study mainly involved very poor verbal STM capacity and occasional phonological paraphasias during speech output (see Table 1). Each patient's performance was compared to that of a control group of healthy adults matched for age, gender and level of education $(N=12)$.

\section{Experimental tasks}

Different STM tasks, all presented auditorily and requiring no overt verbal output, were constructed to maximize the retention of serial order or item information; however none of these tasks should be considered to be a perfectly pure order or item STM task, given that order information is always conditional to item information. The first task was a serial order reconstruction task where digit sequences of increasing length (3-9) were presented; in order to minimize item information processing requirements, item information was known in advance: for a given sequence length, exactly the same items were used and only their order of presentation changed (e.g., for sequence length 4 , the items were repeatedly sampled from the pool $\{1,2,3,4\}$ ); at recall, the participants reconstructed the order of presentation of the digits by arranging cards displaying the presented digits. For the serial order recognition task, word sequences of increasing length (3-7) were presented, the words being again repeatedly sampled from a highly restricted pool of familiar, high frequency words in order to minimize item processing requirements; each trial was immediately followed by a recognition trial, containing the same items but with a serial position exchange for two adjacent items in $50 \%$ of trials.

Tasks maximizing the retention of phonological item information were word or nonword item recognition tasks, each item being new on every trial; the word or nonword items were presented in sequences of increasing length (2-5); each sequence was followed by the re-presentation of all target stimuli and an equal number of distractor stimuli in random order; after each probe stimulus, the participants decided whether the probe stimulus matched 
one of the stimuli in the target list; distractor probe stimuli differed from the target stimulus by a single phoneme; no retention of serial order information was necessary in these tasks.

Table 1: General language and STM profiles of patients TG, AP and DC, and results for the experimental STM tasks maximizing order or item retention requirements

\begin{tabular}{|c|c|c|c|}
\hline & TG & $\mathbf{A P}$ & DC \\
\hline \multicolumn{4}{|l|}{ General language and STM profile } \\
\hline Speech perception ${ }^{a}$ & $94(.93+.01)$ & $.99(.99+.01)$ & $.95(.93+.01)$ \\
\hline Word-picture matching & Perc 79 & $.79(.76+.02)$ & Perc 70 \\
\hline Picture naming & $.88(.94+05)$ & $.87(.94+.05)$ & $.92(.94+.05)$ \\
\hline Sentence comprehension & Perc 50 & Perc 50 & Perc 18 \\
\hline Digit span & $5(5.7+.07)$ & $3^{\mathrm{e}}(5.8+.93)$ & $4^{\mathrm{e}}(5.7+.07)$ \\
\hline Word immediate serial recall $^{\mathrm{b}}$ & $48^{\mathrm{e}}(54+$ & $20^{\mathrm{e}}(52+5.3)$ & $40^{\mathrm{e}}(54+1.8)$ \\
\hline \multicolumn{4}{|l|}{ Experimental STM tasks } \\
\hline Serial order reconstruction ${ }^{c}$ & $22(24.5+3.1)$ & $\mathbf{1 1}^{\mathrm{e}}(18.3+2.7)$ & $1^{\mathrm{e}}(24.5+3.1)$ \\
\hline Serial order recognition ${ }^{\mathrm{d}}$ & $.73(.79+.06)$ & $.57^{\mathrm{e}}(.76+.08)$ & $.55^{\mathrm{e}}(.79+.06)$ \\
\hline Item recognition (words) ${ }^{\mathrm{d}}$ & $.75^{\mathrm{e}}(.86+.04)$ & $.71(.78+.05)$ & $.72^{\mathrm{e}}(.86+.04)$ \\
\hline Item recognition (nonwords) ${ }^{d}$ & $.62^{\mathrm{e}}(.72+.04)$ & $.63(.63+.04)$ & $.65(.72+.04)$ \\
\hline
\end{tabular}

If not otherwise indicated, results show the proportion of correct performance (mean and standard deviations of the control samples are given in parenthesis).

${ }^{a}$ Minimal pair discrimination task for patients TG and DC; phoneme repetition task for patient AP.

${ }^{b}$ Number of words correctly recalled, by pooling over sequence lengths 2 to 5 (4 trials per sequence length)

Number of correct trials, by pooling over all sequence lengths.

${ }^{\mathrm{d}}$ Proportion of correct recognitions, by pooling over all sequence lengths.

${ }^{\mathrm{e}}$ Indicates performance significantly impaired relative to the control group at $p<.05$ (modified $t$-test).

\section{Results and discussion}

We observed selective deficits for item and order STM tasks in two of the patients, TG and AP (see Table 1): TG was significantly impaired for both item STM tasks (mean Z-score: -.2.63), but his performance was preserved for the serial order STM tasks (mean Z-score: -.09); the reverse dissociation was observed for patient AP, with a strong deficit for serial order STM but preserved item STM (mean Z-scores for item and order STM tasks: -0.7 and -2.60, respectively). Patient DC showed a mixed profile with impairments for both serial order and item STM tasks (mean Z-scores for item and order STM tasks: -2.63 and -3.12 , respectively). DCs mixed profile is consistent with his abnormal brain activation in both the temporo-parietal area (supporting item STM, following recent neuro-imaging evidence) and the right intraparietal sulcus (supporting order STM; Majerus et al., 2006a). In contrast, for TG, his selective item STM impairment is consistent with abnormal brain activation restricted to the temporo-parietal areas.

\section{Conclusion}

This study is the first to show a double dissociation between serial order and item short-term storage processes in STM patients. At a theoretical level, these results provide significant support to recent models of STM distinguishing separate mechanisms for item STM and order STM. Recognizing differential patterns of impairment for item and order STM will also have implications for the rehabilitation of short-term storage deficits in STM patients.

\section{References}

Burgess, N., \& Hitch, G. (2006). A revised model of short-term memory and long-term learning of verbal sequences. Journal of Memory and Language, 55, 627-652

Majerus, S., Poncelet, M., Van der Linden, M., Albouy, G., Salmon, E., Sterpenich, V., et al. (2006a). The left intraparietal sulcus and verbal short-term memory: Focus of attention or serial order? NeuroImage, 32, 880-891.

Majerus, S., Poncelet, M., Greffe, C, \& Van der Linden, M. (2006b). Relations between vocabulary development and verbal short-term memory: The relative importance of short-term memory for serial order and item information. Journal of Experimental Child Psychology, 93, 95-119. 